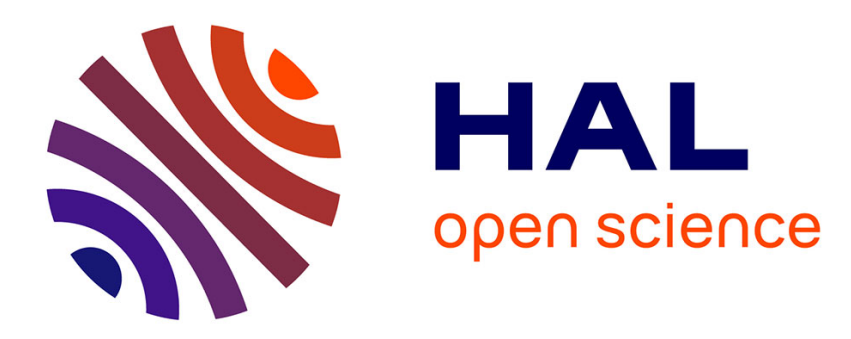

\title{
Extraction of a cyclostationary source using a new cost function without pre-whitening
}

Cécile Capdessus, A.K. Nandi

\section{To cite this version:}

Cécile Capdessus, A.K. Nandi. Extraction of a cyclostationary source using a new cost function without pre-whitening. Signal Processing, 2011, 91 (11), pp.2497-2505. hal-00607037

\section{HAL Id: hal-00607037 \\ https://hal.science/hal-00607037}

Submitted on 8 Jul 2011

HAL is a multi-disciplinary open access archive for the deposit and dissemination of scientific research documents, whether they are published or not. The documents may come from teaching and research institutions in France or abroad, or from public or private research centers.
L'archive ouverte pluridisciplinaire HAL, est destinée au dépôt et à la diffusion de documents scientifiques de niveau recherche, publiés ou non, émanant des établissements d'enseignement et de recherche français ou étrangers, des laboratoires publics ou privés. 
Extraction of a cyclostationary source using a new cost function without prewhitening

\section{Authors}

Cécile CAPDESSUS, Corresponding author

Institut PRISME, Université d'Orléans

Postal address :

21 rue Loigny la Bataille, 28000 CHARTRES, France

Tel : +33 (0)2 37333838 or $+33(0) 237918320$

Fax : +33(0)2 37918329

e-mail : cecile.capdessus@univ-orleans.fr

Asoke K. NANDI

Department of Electrical Engineering \& Electronics

The University of Liverpool

Brownlow Hill, Liverpool, L69 3GJ, UK

Tel: +44151794 4525

Fax: +44 1517944540

e-mail : a.nandi@liverpool.ac.uk 


\begin{abstract}
The proposed method aims to extract a cyclostationary source, whose cyclic frequency is a priori known, from a set of additive mixtures. The other sources may be either stationary or cyclostationary as long as their cyclic frequencies are different from that of the source to be extracted. The method does not require pre-whitening and consists in minimising a criterion based on stationary and cyclostationary second order statistics of the observations; this method is labeled as Second Order Cyclostationary Statistics Optimization Criterion $\left(\mathrm{SOC}^{2}\right)$. The relevance of this criterion is proven theoretically in the general case of $N$ sources by $P$ sensors, with $P \geq N$. Other properties of the algorithm such as its accuracy and its robustness against additive noise or strong interferences are studied through a set of simulations.
\end{abstract}

\title{
Keywords
}

Source extraction, second order statistics, cyclostationarity. 
Source separation has been widely developed over the last decades, due to its potential applications in many domains, including biomedical and communications. It consists in retrieving some information called sources from a set of observations which are distinct mixtures of these sources. The mixtures can be supposed to be additive or convolutive, depending on the physical nature of the application. When this separation is performed without any knowledge about either the sources or the propagation paths, it is called blind. Some semi-blind methods have also been developed, taking advantage of a priori knowledge about the sources, for instance about their probability density function or non-stationary properties. In some applications there is no need to separate all the sources. When only one of the sources is of interest (SOI) and the other ones are considered as interferences to be removed, it leads to the source extraction problem. The method that we propose aims to extract a cyclostationary source whose cyclic frequency is a priori known from a set of additive mixtures.

In various domains, such as telecommunications and vibration analysis of rotating machines, most signals exhibit cyclostationarity, for instance at the baud rate of the communication signal or the frequency of its carrier. If known, this information can be used in order to improve the classical source separation or extraction methods or to propose new ones. So far, different strategies have been chosen, which consist in either trying to apply stationary methods to cyclostationary signals, or developing new methods specifically meant to be applied to cyclostationary ones. Among these methods, some suppose that all the sources share a common cyclic frequency, others are based on the opposite hypothesis, i.e. that they all exhibit different cyclic frequencies, or can cope with either case.

Different techniques have been developped in the additive mixture context. Ferréol and Chevalier [1], and later Ferréol et al. [2] studied the influence of cyclostationarity on classical methods developed for within the stationary framework such as SOBI and fourth order cumulant based methods and showed that, if not taken into account, the cyclostationarity of the sources can lessen their performance. Later, Jallon and Chevreuil [3] studied the effect of cyclostationarity on Comon contrast function [4]. They showed that it still is a contrast in the presence of some specific cyclostationary sources, though estimated on stationary hypotheses, and proposed an extension that can be used when the cyclic frequencies of the sources are known. When all the sources share a common cyclic frequency, Boustany and Antoni $[5,6]$ and Antoni et al. [7] proposed two different methods. One is a modified version of SOBI called CYCLOSOBI, which consists in jointly diagonalising cyclic correlation matrices, while the other one uses a subspace decomposition of the observations via their cyclic statistics. A related method was proposed by Rhioui et al. [8], based on second order cyclic temporal moments. Ghaderi et al. [9] proposed an extraction method for a set of sources sharing the same cyclic frequency. 
More methods were developed in the case where the sources exhibit distinct cyclic frequencies. Jafari et al. [10] improved the Kullback-Leibler divergence minimisation criterion by adding a term consisting of the distance between the cyclic covariance matrix of the estimates and the identity matrix. Liang et al. [11] also proposed to use cyclic correlation matrices but for different time lags. Houcke et al. [12] developed a deflation method which uses the cyclostationary properties of the sources through a prior resampling. Abed Meraim et al. [13] developed a contrast function based on the second order temporal statistics of the sources, both stationary and cyclostationary, and they introduce a modified version aiming at the extraction of one specific source. Xiang [14] as well as Keziou and Ould Mohamed [15] also built a cost function from second order cyclic statistics. Liang et al. [16] as well as Wang et al. [17] propose methods based on singular values decomposition of cyclic covariance matrices. Liang et al. [18] proposed a method based on a network whose coefficients are refreshed from the cyclic statistics of the estimate.

Agee et al. [19] developed a family of cyclostationarity based source extraction methods using a property restoral approach which led them to propose three different SCORE (Self COherence REstoral) algorithms. Two of them consist in comparing the estimate to a frequency shifted version of the observation. The third one, called direct SCORE algorithm, consists in maximising the self-coherence of the estimate at the cyclic frequency of the source to be extracted. It turns out that the method that we propose here is a new way of interpreting and implementing this property restoral approach. In [20], Bouguerriou et al. propose a method based on prior whitening and then a simple cost function based on the cyclic statistics of the source to extract, while Boustany and Antoni [21] propose a subspace decomposition. Ghaderi et al. [22] implement an iterative diagonalisation method of the cyclic covariance matrix estimated at the cyclic frequency of the SOI and Xiang [23] minimises a cyclic statistics based cost function with a constraint on the power of the estimate. Preliminary versions of the method presented here have appeared previously in [24, $25]$ and an application to ECG signals was presented in [25, 26].

In some applications such as rotating machinery monitoring or ECG signals analysis, it could be interesting to implement real time extraction of a cyclostationary source on embedded systems. We aimed at designing an algorithm that could be implemented easily in real time processors, which means that complex and heavy calculations such as higher order statistics, eigenvalue decompositions and matrix inversions should be avoided, as well as tricky parameter choice. Our extraction algorithm is thus based only on second order statistics calculations and matrix products, which can easily be handled with any real-time processor device. No parameter must be chosen since the optimization algorithm computes automatically the optimal step. As will be shown later, its performances are at least equivalent to and often better than those of the main algorithms that have been developed so far for the extraction of a cyclostationary source. The proposed method will be referred to as Second Order Cyclostationary Statistics Optimization Criterion $\left(\mathrm{SOC}^{2}\right)$ through the rest of the paper. 
The problem is stated in section 2. In section 3, we present the extraction method and prove theoretically that it leads to the extraction of the SOI. In section 4 we formulate the optimization problem and the method used for the simulations is described. Section 5 presents some simulations performed in order to enhance the main features and performances of our algorithm. It is compared through these simulations to Abed-Meraim's ATH3 algorithm [13] and Agee's phase-SCORE [19] algorithm and the presented plots are discussed. Section 6 gives a conclusion and some perspectives of further developments. 


\section{Problem statement}

Here we use the classical formulation for source separation. A set of $P$ sensors receive simultaneously $N$ source signals put together in a so called source vector $\mathbf{s}(t)=\left[s_{1}(t) s_{2}(t) . s_{N}(t)\right]^{T}$ where $t$ stands for the continuous time and $T$ for the transpose operator. The $P$ observations are put together in an observation vector $\mathbf{x}(t)=\left[\begin{array}{lll}x_{1}(t) x_{2}(t) . \quad x_{P}(t)\end{array}\right]^{T}$ that can be expressed using the source vector and a mixing matrix $\mathbf{A}$ in the following way:

$\mathbf{x}(t)=\mathbf{A s}(t)$

In what follows the sources as well as the observations will be supposed to be real signals and the mixing matrix to be a real $P$ by $N$ matrix. This corresponds to a case where the observations are free from any noise. The noisy case will be commented further within the present section and studied later using the simulations. We suppose without any loss of generality that the source of interest (SOI) is $s_{1}(t)$.

The hypotheses are the following ones :

- The SOI is second order cyclostationary at a frequency $\alpha_{0}$ a priori known.

- All the other sources can be either stationary or cyclostationary, provided that none of them is cyclostationary at the same frequency as $s_{1}(t)$.

- All sources are zero mean and uncorrelated so that their joint second order stationary as well as cyclostationary statistics are equal to zero.

- The mixing matrix is full rank.

- The number of sensors $P$ is greater or equal to the number of sources $N$.

Extracting the source $s_{1}(t)$ consists in estimating a b row vector of $P$ coefficients such that $z(t)=\mathrm{b} \mathbf{x}(t)$ is an estimate of $s_{1}(t)$.

\section{Extraction method}

Let us define the statistics that are used to specify the criterion. Given a zero-mean real-valued signal, $s(t)$, its covariance $r_{s}$ and cyclic covariance $r_{s}^{\alpha}$ at frequency $\alpha$ can be defined as:

$r_{s}=\lim _{\theta \rightarrow \infty}\langle s(t) s(t)\rangle_{\theta}$

$r_{s}^{\alpha}=\lim _{\theta \rightarrow \infty}\left\langle s(t) s(t) e^{-2 \pi j \alpha t}\right\rangle_{\theta}$

The brackets \langle\rangle$_{\theta}$ denote temporal averaging over $\theta$ seconds. 
Given a column vector $\mathbf{s}(t)$ of $\mathrm{P}$ zero-mean real-valued components, $s_{i}(t)$, its covariance matrix $\mathbf{R}_{s}$ and cyclic covariance matrix $\mathbf{R}_{s}^{\alpha}$ at frequency $\alpha$ can be defined as:

$\mathbf{R}_{\mathbf{s}}=\lim _{\theta \rightarrow \infty}\left\langle\mathbf{s}(t) \mathbf{s}^{T}(t)\right\rangle_{\theta}$

$\mathbf{R}_{\mathbf{s}}^{\alpha}=\lim _{\theta \rightarrow \infty}\left\langle\mathbf{s}(t) \mathbf{s}^{T}(t) e^{-2 \pi j \alpha t}\right\rangle_{\theta}$

In order to extract the cyclostationary source at a given frequency, the estimate should be as cyclostationary as possible at that frequency while the power of the estimate is minimized so as to suppress any interference that is not cyclostationary at the same frequency. We thus propose to minimize the ratio between the power of the estimate, given by $\mathbf{b} \mathbf{R}_{\mathbf{x}} \mathbf{b}^{T}$, and its cyclic power, given by $\left|\mathbf{b} \mathbf{R}_{\mathbf{x}}^{\alpha_{0}} \mathbf{b}^{T}\right|$.

\section{III-1 The free from noise case}

The $\mathrm{SOC}^{2}$ method then consists of minimizing over $\mathbf{b}$ the following criterion:

$c(\mathbf{b})=\left|\frac{\mathbf{b} \mathbf{R}_{\mathbf{x}} \mathbf{b}^{T}}{\mathbf{b} \mathbf{R}_{\mathbf{x}}^{\alpha_{0}} \mathbf{b}^{T}}\right|$

where $\mathbf{R}_{\mathbf{X}}$ is the $P$ by $P$ covariance matrix of the observations and $\mathbf{R}_{\mathbf{X}}^{a_{0}}$ is the $P$ by $P$ cyclic covariance matrix of the observations at frequency $\alpha_{0}$. It should be noted that the covariance matrices are calculated for a zero time-lag, so that the algorithm can apply only to the extraction of a source that exhibits non zero cyclic correlation at that time lag, which is not the case for all cyclostationary sources.

First proposition : this criterion admits an absolute minimum as a function of $\mathbf{b}$.

Proof

The criterion can be written as a function of the $N$ by $N$ covariance matrix and the $N$ by $N$ cyclic covariance matrix of the source vector as follows.

$$
c(B)=\left|\frac{\mathbf{b} \mathbf{A} \mathbf{R}_{\mathbf{S}} \mathbf{A}^{T} \mathbf{b}^{T}}{\mathbf{b} \mathbf{A} \mathbf{R}_{\mathbf{S}}^{\mathbf{a}_{0}} \mathbf{A}^{T} \mathbf{b}^{T}}\right|
$$

Since the sources are zero mean and uncorrelated, the covariance matrix of the source vector is a diagonal one, whose diagonal terms are the powers of the sources. Let us denote the power of the $i^{\text {th }}$ source by $\tau_{i}{ }^{2}$, the $i^{\text {th }}$ coefficient of the vector $\mathbf{b}$ by $b_{i}$ and the coefficient on the $i^{\text {th }}$ row and the $j^{\text {th }}$ column of $\mathbf{A}$ by $a_{i}$. Then the numerator $n(\mathbf{b})$ of the criterion can be expressed as

$$
n(\mathbf{b})=\sum_{j=1}^{N} \sigma_{j}^{2} e_{j}^{2}(\mathbf{b})
$$


with

$$
e_{j}(B)=\sum_{i=1}^{P} b_{i} a_{j}
$$

Since only the source $s_{1}(t)$ is cyclostationary at frequency $\alpha_{0}$, the cyclic covariance matrix exhibits only one non zero coefficient, that is to say

$$
\mathbf{R}_{\mathbf{S}}^{\alpha_{0}}=\left[\begin{array}{ccc}
r_{s_{1}}^{\alpha_{0}} & \ldots & 0 \\
\vdots & & \vdots \\
0 & \ldots & 0
\end{array}\right]
$$

where $r_{s_{1}}^{\alpha_{0}}$ is the cyclic covariance of the source $s_{1}(t)$ at frequency $\alpha_{0}$ and for zero time lag. The criterion now simplifies to

$$
c(\mathbf{b})=\left|\frac{1}{r_{s_{1}}^{\alpha_{0}}}\left(\sigma_{1}^{2}+\frac{\sum_{j=2}^{N} e_{j}^{2}(\mathbf{b}) \sigma_{j}^{2}}{e_{1}^{2}(\mathbf{b})}\right)\right|
$$

The criterion reaches an absolute minimum for any value of $\mathbf{b}$ such that $\mathbf{e}=\left[e_{1}(\mathbf{b}) e_{2}(\mathbf{b}) \ldots e_{P}(\mathbf{b})\right]=[e 0 \ldots 0]$ with $e \neq 0$

The vector $\mathbf{e}$ can be expressed as $\mathbf{e}=\mathbf{b} \mathbf{A}$. So, provided that $\mathbf{A}$ is full rank, the linear equations system described by $\mathbf{b} \mathbf{A}=\left[\begin{array}{lll}e & 0 & \ldots\end{array}\right]$ can be solved. Since there exist an infinity of possible non zero values for $e$ there exist as well an infinity of corresponding $\mathbf{b}$ vectors which minimize the criterion.

Second proposition : any value of the vector $\mathbf{b}$ that makes the criterion reach its minimum leads to the extraction of the source $s_{1}(t)$.

Proof

Let us express the estimate $z(t)$ as a function of the sources.

$$
z(t)=\mathbf{b} \mathbf{A} \mathbf{s}(t)=\sum_{j=1}^{N} \boldsymbol{e}_{j}(\mathbf{b}) \boldsymbol{s}_{j}(t)
$$

For any vector $\mathbf{b}_{\min }$ that minimizes the criterion, all $\boldsymbol{e}_{j}\left(\mathrm{~b}_{\min }\right)$ are zero except for $e_{1}\left(\mathrm{~b}_{\min }\right)$. Thus $z(t)=e_{1}\left(\mathrm{~b}_{\min }\right) s_{1}(t)$ is an estimate of the source $s_{1}(t)$ with an indeterminacy on the amplitude, as in any source separation or extraction method. This holds for the case where the number of sensors is equal to the number of sources $(P=N)$ as well as in the over-determined case $(P>N)$ which are the 
hypotheses that we made in section 2. But in the under determined case $(P<N)$ minimizing the criterion does not lead any more to the extraction of $s_{1}(t)$. Indeed the equation $\mathbf{b}=\left[\begin{array}{lll}e & 0 & \ldots\end{array}\right]$ cannot be solved since there are more equations than $\mathbf{b}$ coefficients.

\section{III-2 The noisy case}

In the noisy case, the model becomes

$$
\mathbf{x}(t)=\mathbf{A} \mathbf{s}(t)+\mathbf{w}(t)
$$

where $\mathbf{w}(t)=\left[w_{1}(t) w_{2}(t) \ldots w_{p}(t)\right]^{T}$ represents the additive noise that are received on the sensors with the mixture of the sources. Let us suppose that the noise components are stationary and denote by $\mathbf{R}_{\mathrm{w}}$ the noise vector covariance matrix. The expression of the criterion then becomes

$$
c(\mathbf{b})=\left|\frac{1}{r_{\mathbf{s}_{1}}^{\alpha_{0}}}\left(\sigma_{1}^{2}+\frac{\sum_{j=2}^{N} e_{j}^{2}(\mathbf{b}) \sigma_{j}^{2}+\mathbf{b} \mathbf{R}_{\mathbf{w}} \mathbf{b}^{T}}{e_{1}^{2}(\mathbf{b})}\right)\right|
$$

If the noise components $w_{j}(t)$ are correlated to each other, then the term $\mathbf{b} \mathbf{R}_{\mathbf{w}} \mathbf{b}^{T}$ can be positive as well as negative, so that the proof given in section 2 does not hold any more and one can suppose that minimizing the criterion in that case does not achieve the extraction.

If the noise components are uncorrelated to each other, the numerator of the criterion is modified by an additive positive term depending on $\mathbf{b}$, which might as well alter the method. The effect of additive, uncorrelated noise will be studied from simulations.

\section{Implementation}

\section{IV -1 Algorithm}

The criterion is minimized by a Fletcher-Reeves algorithm as described in [28].

Fletcher-Reeves algorithm is well-adapted to the optimization of non linear functions. Furthermore, it can be applied without any prior choice of a step size, since the optimal step direction $\mathrm{d}_{\mathrm{k}}$ and step size $\alpha_{k}$ are calculated at each step from the gradient and Hessian of the criterion. These are calculated by using the expressions 
derived respectively in appendix $A$ and appendix $B$. Each new direction is chosen to be orthogonal to the previous one. After every $P$ step, the algorithm starts again with an initial direction computed from the gradient.

The successive steps are :

1. Estimate the covariance matrix $\mathbf{R}_{\mathrm{X}}$ and the cyclic covariance matrix $\mathbf{R}_{\mathrm{x}}^{\mathrm{a}_{0}}$ from the observations. Chose a tolerance value $\varepsilon$.

2. Choose an arbitrary $\mathbf{b}_{0}$ initial $\mathbf{b}$ vector and compute the initial gradient $\mathbf{g}_{0}$. Use it as a first descent direction by setting $\mathbf{d}_{0}=-\mathbf{g}_{0}$. Set index $k=0$.

3. Compute the optimal step size $\alpha_{k}=-\frac{d_{k}{ }^{T} g_{k}}{d_{k}{ }^{T} H_{k} d_{k}}$ where $H_{k}$ is the Hessian of the criterion computed at the $k^{\text {th }}$ step (see Appendix B). Compute the new extraction vector from the previous one by $\mathbf{b}_{k+1}=\mathbf{b}_{k}+\alpha_{k} \mathbf{d}_{\mathbf{k}}$.

4. If $\left\|\alpha_{k} \mathbf{d}_{\mathbf{k}}\right\|<\varepsilon$, output $\mathbf{b}_{\text {min }}=\mathbf{b}_{k+1}$ and stop.

5. If $k=P-1$, set $\mathbf{b}_{0}=\mathbf{b}_{k+1}$ and go to step 2 .

6. Compute the new gradient value $\mathbf{g}_{k+1}$ (see Appendix A) and compute the new orthogonal direction $d_{k+1}=-g_{k+1}+\beta_{k} d_{k}$ with $\beta_{k}=\frac{g_{k+1}{ }^{T} g_{k+1}}{g_{k}^{T} g_{k}}$. Refresh the index $k=k+1$ and repeat from step 3 .

Fletcher-Reeves algorithm is based on an approximation made on the Taylor series decompositions of the criterion and its gradient when close to the minimum. Thus it is not valid when the starting point is far from the minimum of the criterion and would never converge in this case. In order to overcome this problem two precautions are used :

- First, the initial $\mathbf{b}$ vector is chosen from among ten random ones as the one which minimizes the criterion.

- Second, the initial extraction vector $\mathbf{b}_{0}$ is tested when the algorithm reaches step 3. The criterion is computed with the new extraction vector $\mathbf{b}_{k+1}$ and if its value is lower than the initial one, the program goes on. If its value is on the contrary greater than the initial one, the program is started again with another random initial extraction vector.

\section{IV-2 Estimation of the covariance matrices}


The cyclic covariance matrix is estimated by temporal averaging over $N_{r}$ realizations of the cyclostationary SOI, i.e. over $N_{r} / \alpha_{0}$ seconds. The same set of data is used to estimate the covariance matrix of the observations.

That is to say:

$\left[\begin{array}{c}\widehat{\boldsymbol{R}}_{\boldsymbol{x}}=\left\langle\boldsymbol{x}(t) \boldsymbol{x}^{T}(t)\right\rangle_{N_{r} / \alpha_{0}} \\ \widehat{\boldsymbol{R}}_{\boldsymbol{x}}^{\alpha_{0}}=\left\langle\boldsymbol{x}(t) \boldsymbol{x}^{T}(t) \boldsymbol{e}^{-2 \pi j \alpha_{0} t}\right\rangle_{N_{r} / \alpha_{0}}\end{array}\right.$

The brackets \langle\rangle$_{\theta}$ denote temporal averaging over $\theta$ seconds.

IV - 2 Performances evaluation

In order to evaluate the performance of the $\mathrm{SOC}^{2}$ algorithm, a performance index, inspired by the formulations in [10] and [13], will be computed for all simulations. It is given by

$P I=\frac{\sum_{k=2}^{N}\left|w_{k}\right|^{2}}{\left|w_{1}\right|^{2}}$

with $\mathbf{w}=\mathbf{b} \mathbf{A}$ and $w_{k}$ is the $\mathbf{k}^{\text {th }}$ coefficient of vector $\mathbf{w}$.

For some simulations it will be given in $\mathrm{dB}$ as

$P I_{d B}=10 * \log _{10}(P I)$

The performance index is the classical measure for the efficiency of a separation or extraction method. It estimates the ratio between the interferences and the SOI, so that the smallest it is the best is the extraction.

Also for some experiments we will plot the mean squared error between the estimate and the SOI. The estimate as well as the SOl's amplitudes are normalized prior to the error computation. 


\section{Numerical simulations}

In all the simulations, frequencies are normalized by the sampling frequency and thus given without unit. The simulations presented in sections V-1 to V-4 have some common features that are described here. There are $N=2$ sources and $P=2$ sensors. Both the sources are cyclostationary at different cyclic frequencies. These two sources are cosine waves amplitude modulated by colored noise. The carrier frequency of the source $s_{1}(t)$ is $f_{1}=0.05$. The modulating signal is a Gaussian random white noise filtered by a $4^{\text {th }}$ order Butterworth low-pass filter whose cut-off frequency is $f_{c}=0.0175$. The carrier frequency of the source $s_{2}(t)$ is $f_{2}=0.04$. The modulating signal is a Gaussian random white noise filtered by a $4^{\text {th }}$ order Butterworth low-pass filter whose cut-off frequency is $f_{c 2}=0.0185$. They are both cyclostationary at twice their carrier frequencies. The frequency used for the extraction will be that corresponding to $s_{1}(t)$, that is to say $\alpha_{0}=2^{*} f_{1}$. Unless otherwise stated, the two sources have unit power.

The $\mathrm{SOC}^{2}$ method is compared to two other cyclostationary source extraction methods: Abed-Meraim's ATH3 algorithm [13] and Agee's phase-SCORE [19]. Both methods are based on an algebraic estimation of the extraction vector.

In ATH3 algorithm, the extraction vector is estimated as

$$
\mathrm{b}=\boldsymbol{R}^{-H / 2} \boldsymbol{c}_{\boldsymbol{i}}
$$

where $\boldsymbol{c}_{\boldsymbol{i}}$ is the least eigenvector of $\boldsymbol{R}^{-1 / 2} \widetilde{\boldsymbol{R}}_{\boldsymbol{i}} \boldsymbol{R}^{-H / 2}$ and (.) ${ }^{H}$ is the Hermitian conjugate, with the $\boldsymbol{R}$ matrix and $\boldsymbol{R}_{\boldsymbol{i}}$ matrix defined as follows:

$\boldsymbol{R} \stackrel{\text { def }}{=} \lim _{\theta \rightarrow \infty}\left\langle\boldsymbol{x}(t) \boldsymbol{x}^{H}(t) \sum_{j=1}^{m} e^{j \alpha_{j} t}\right\rangle_{\theta}$

$\widetilde{\boldsymbol{R}}_{\boldsymbol{i}} \stackrel{\text { def }}{=} \lim _{\theta \rightarrow \infty}\left\langle\boldsymbol{x}(t) \boldsymbol{x}^{H}(t) \sum_{j \neq i} e^{j \alpha_{j} t}\right\rangle_{\theta}$

with $\alpha_{i}$ the cyclic frequency of the source to be extracted and $\left\{\alpha_{j}, j \neq i\right\}$ the cyclic frequencies of the other sources. The hypotheses about the sources are that they all are cyclostationary and their cyclic frequencies are a priori known.

In phase-SCORE algorithm, the extraction vector is the eigenvector corresponding to the greatest eigenvalue of the matrix

$$
\left(\boldsymbol{R}_{x x}\right)^{-1} \boldsymbol{R}_{x x}^{\alpha_{0}}(\tau)
$$


In our case we will apply it with a zero time-lag, so that we will compute the eigenvector corresponding to the greatest eigenvalue of

$$
\left(R_{x x}\right)^{-1} R_{x x}^{\alpha_{0}}
$$

This algorithm is based on the same hypothesis as ours and uses the same covariance matrices, i. e. the stationary covariance matrix of the observation vector and its cyclic covariance matrix at the frequency of the source to extract.

In section $\mathrm{V}-1$ the robustness of the $\mathrm{SOC}^{2}$ algorithm is tested relative to the number of samples used for the estimation of the covariance matrices. In section V-2 its robustness is tested relative to the mixing matrix. The robustness of the algorithm relatively to the power of the interference, i.e. the source, $s_{2}(t)$, is explored in section V-3, while in section V-4 the same relative to the presence of additive noise is studied. In section V-5 a 3 by 3 experiment is presented. In all the experiments, the tolerance for the optimization algorithm has been chosen to be $\varepsilon=10^{-10}$.

\section{V-1 Robustness relative to the number of samples}

The mixing matrix is fixed and chosen to be a well conditioned one :

$$
\mathbf{A}=\left[\begin{array}{cc}
2.3829 & 0.7311 \\
-0.6986 & 1.2667
\end{array}\right]
$$

The number of samples used for the estimation of the covariance matrices varies from 200 to 8000 in steps of 200 samples, so that the number of cycles of the source $s_{1}(t)$ varies from 10 to 40 in steps of 10 cycles. For each number of samples, 1000 experiments are carried out with different sources and all the results are averaged over these 1000 experiments. The results obtained for the three methods are plotted in Fig. 1. Whatever number of samples is used for the estimation of the covariance matrices, the $\mathrm{SOC}^{2}$ algorithm shows better performances than the other two. 


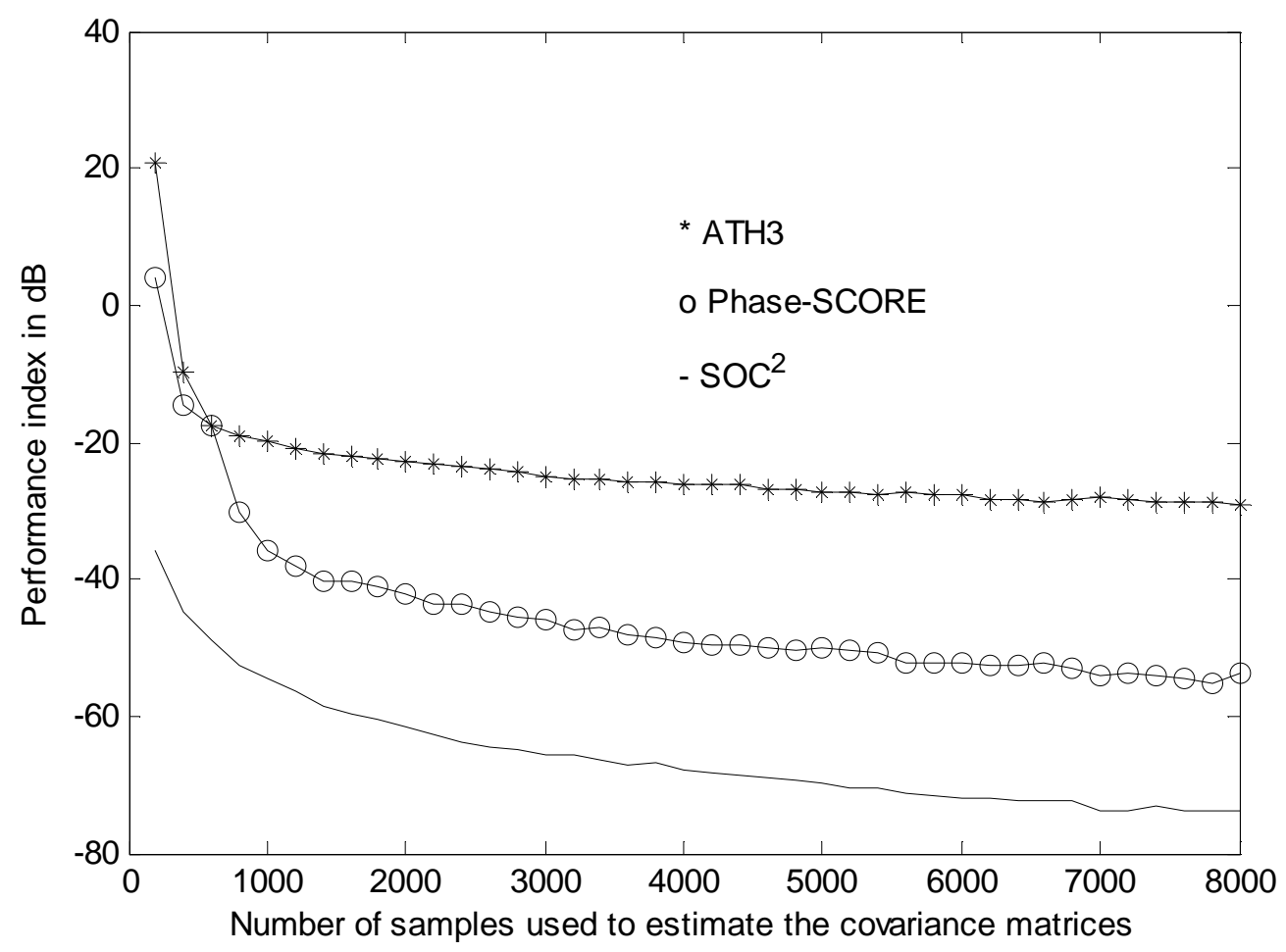

Fig 1

Performance Index in $\mathrm{dB}$ versus the number of samples used to estimate the covariance matrices.

\section{V-2 Robustness relative to the mixing matrix}

In this experiment 1000 well conditioned random mixing matrices are tested. The two sources are the same for all the experiments. The averaged PI and its standard deviation are computed over the 1000 experiments. The results obtained for the three different methods are summarized in Table 1. The SOC ${ }^{2}$ algorithm achieves extraction with better accuracy than the other two, independent on the mixing matrix.

\begin{tabular}{|l|l|l|l|}
\hline & ATH3 & Phase-SCORE & SOC $^{2}$ \\
\hline Average PI & $13.0^{*} 10^{-4}$ & $2.6^{*} 10^{-6}$ & $3.5^{*} 10^{-8}$ \\
\hline Standard deviation & $5.2^{*} 10^{-4}$ & $2.4^{*} 10^{-6}$ & $0.0^{*} 10^{-8}$ \\
\hline
\end{tabular}

Table 1

Average performance index and its standard deviation computed over 1000 experiments carried out with the same sources and different random mixing matrices. 


\section{V-3 Performance relative to the power of the interference.}

Here the power of the source $s_{2}(t)$ varies $15 d B$ from to $-5 d B$ in steps of $2 d B$, so that the signal to interference ratio (SIR) varies from $-15 d B$ to $5 d B$ in steps of $2 d B$. For each value of the SIR the $\mathrm{PI}$ is averaged over 1000 experiments carried out with different sources and a fixed mixing matrix equal to that used in section $\mathrm{V}-1$. The number of samples is 2000 for all the trials. The PI is plotted versus the SIR for the three methods on Fig 2, and the mean square error is plotted on Fig. 3.

The estimated source is normalized in amplitude prior to the error calculation. Then the squared error between the normalized estimate and the normalized true source is averaged over the 2000 samples. This error is then averaged over the 1000 trials for each value of the SIR.

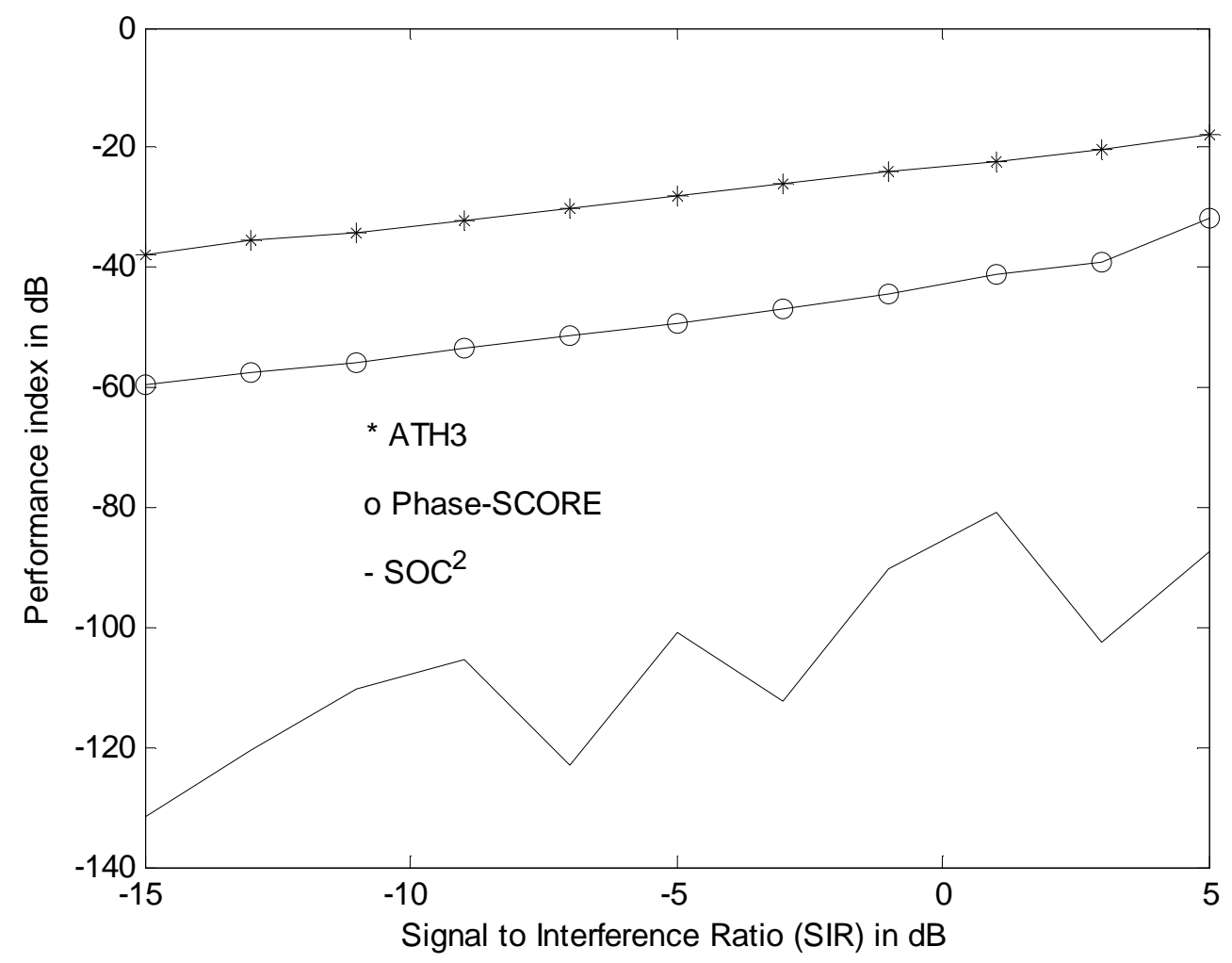

Fig. 2

Performance Index in $\mathrm{dB}$ plotted for the three methods versus the Signal to Interference Ratio. 


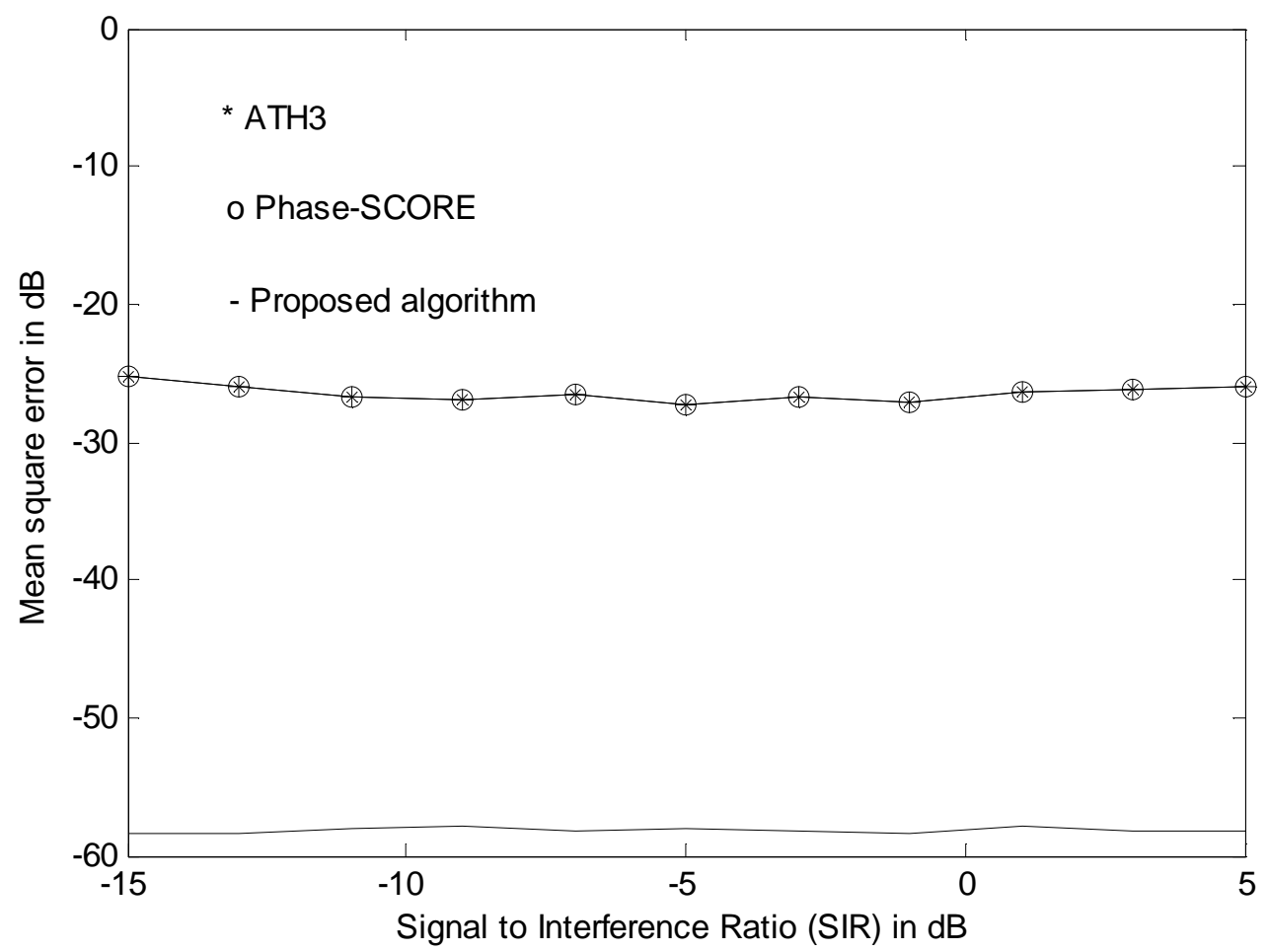

Fig. 3

Mean square error in $\mathrm{dB}$ plotted for the three methods versus Signal to Interference Ratio, averaged both in time and over 50 experiments.

It is interesting to note that the mean square error is fairly constant whatever the power of the interference for each of these three methods, while the performance index improves at low SIR. If normalized by the power of the sources, the performance index would be fixed. Indeed, the performance index is decreased by $20 \mathrm{~dB}$ while the power of the interference is decreased by $20 \mathrm{~dB}$ as well. The two algorithms ATH3 and Phase-SCORE often lead to exactly the same solution, which can be explained by the fact that they both use the inverse of the covariance matrix of the observations, so that the result is tied to the quality of this inversion. The overall conclusion is that the $\mathrm{SOC}^{2}$ method performs significantly better than the two compared methods in terms of both the mean squared error and the performance index.

\section{V-4 Performances in the presence of additive noise}

In these experiments, some temporal and spatially white Gaussian random noise is added to the observations. The signal to noise ratio (SNR) varies from $-40 d B$ to 
$40 d B$ in steps of $2 d B$. For each value of the SNR the PI is averaged over 1000 experiments carried out with different additive noise. The same sources and the same mixing matrix, equal to $A=[0.721 .58 ;-1.6-1.44]$, are used for all the trials. The number of samples is 1000 . The PI is plotted versus the SNR on Fig 4.

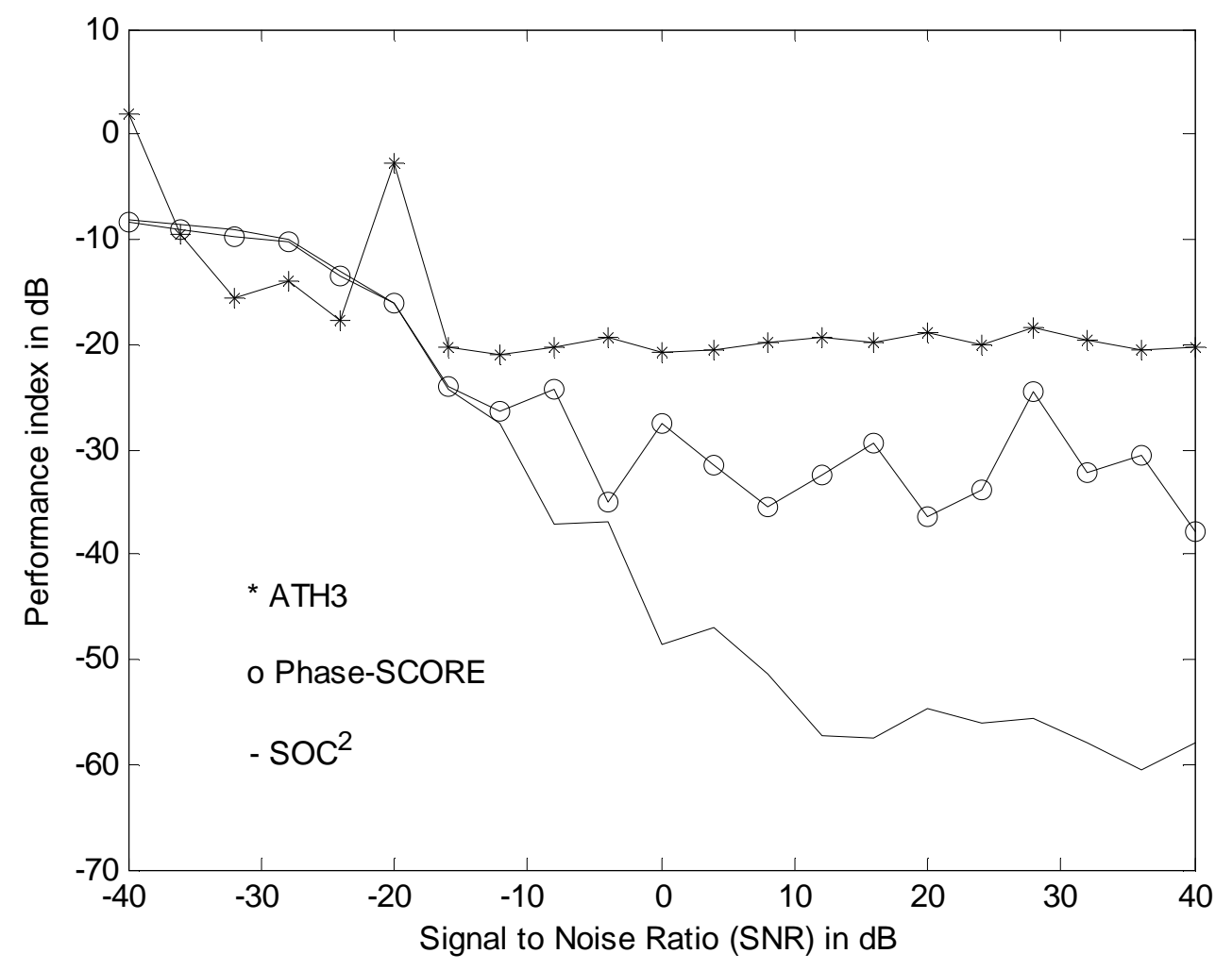

Fig 4

The PI is plotted versus the Signal to Noise Ratio (SNR)

It can be noted from this plot that in very low SNRs all three methods have about the same performance, but for higher SNRs $(>0 \mathrm{~dB})$ the $S \mathrm{CC}^{2}$ algorithm offers much better source extraction (well over a $20 \mathrm{~dB}$ improvement in $\mathrm{Pl}$ ).

\section{V-5 3 by 3 case}

In these investigations, simulations are performed with 3 sources and 3 sensors. The first two sources are the same as in the previous section and the third one is chosen 
the same way with a carrier frequency $f_{3}=0.03$ modulated by a colored noise generated through a Butterworth low-pass filter of cut-off frequency $f_{c 3}=0.018$. The cyclic frequency used for the extraction is $\alpha_{0}=2 * f_{1}$. In the two following experiments, 100 trials were performed with different source and a fixed mixing matrix:

$\boldsymbol{A}=\left[\begin{array}{ccc}0.4927 & 1.2797 & -0.9417 \\ 0.6393 & 0.063 & 1.1563 \\ 0.9172 & 0.4807 & -0.3473\end{array}\right]$.

The averaged $\mathrm{PI}$ and its standard deviation are recorded in Table 2 for the three methods.

\begin{tabular}{|l|l|l|l|}
\hline & ATH3 & Phase-SCORE & SOC $^{2}$ \\
\hline Average PI & 0.02 & $1.2^{*} 10^{-4}$ & $7.0^{*} 10^{-1}$ \\
\hline Standard deviation & 0.02 & $2.7^{*} 10^{-4}$ & $12.5^{*} 10^{-7}$ \\
\hline
\end{tabular}

Table 2

Average performance index and its standard deviation computed over 100 experiments carried out with different sources but the same mixing matrix for 3 sources and 3 sensors.

\section{V-6 Discussion}

It can be seen from the simulations that the $\mathrm{SOC}^{2}$ method exhibits better performances than the ATH3 and phase-SCORE algorithms. In the presence of much additive noise or strong interference (negative SNR or SIR) the performances are about the same but for good SNR and good SIR, the method that we propose achieves the extraction with much better accuracy. This can be explained by the fact that it is based only on very simple calculations such as matrix products whereas the other two methods imply matrix inversion and eigenvalue decomposition, which can lead to numerical errors. It is interesting to note that though the ATH3 algorithm uses more a priori information than the other two, since it needs the knowledge of all the cyclic frequencies of the sources, its performances are poorer. It can come from the fact that more subtle matrix calculations are required, such as the inverse of the covariance matrix which is used several times. These results are also verified in the 3 by 3 case presented in the last section. 


\section{Conclusion}

We have introduced a new source extraction algorithm based on the cyclostationary properties of the source to be extracted. The only information that needs to be known is the value of one cyclic frequency that the source of interest possesses and does not share with any interference. The $\mathrm{SOC}^{2}$ extraction criterion is built from the covariance matrix and the cyclic covariance matrix of the observations. The theoretical proof has been given that the proposed algorithm is relevant for the extraction of the source of interest in the case where there are at least as many sensors as sources. Some simulations bring further information about its behaviour in the presence of strong interferences or additive noise. It turns out to be a powerful tool to extract a cyclostationary source received along with stationary or cyclostationary interferences and the performances of the proposed method have been proven to outperform those of similar methods such as Agee's phase-SCORE algorithm and Abed Meraim's ATH3 extraction algorithm. In all the cases examined the $\mathrm{SOC}^{2}$ algorithm is found to be as good as the aforementioned two algorithms and it often leads to better source extractions. 


\section{Appendix A}

\section{Gradient of the criterion}

$c(\mathbf{b})=\left|\frac{c_{1}(\mathbf{b})}{c_{2}(\mathbf{b})}\right|$

where $c_{1}(\mathbf{b})=\mathbf{b} \mathbf{R}_{\mathbf{X}} \mathbf{b}^{T}$ is a real valued positive function

while $c_{2}(\mathbf{b})=\mathbf{b} \mathbf{R}_{\mathbf{x}}^{\mathbf{a}_{0}} \mathbf{b}^{T}$ is a complex valued function

$c(\mathbf{b})=\sqrt{\left|\frac{c_{1}(\mathbf{b})}{c_{2}(\mathbf{b})}\right|^{2}}$

$\frac{\delta c(\mathbf{b})}{\delta \mathbf{b}}=\frac{1}{2 c(\mathbf{b})} \frac{\delta}{\delta \mathbf{b}}\left(\left|\frac{c_{1}(\mathbf{b})}{c_{2}(\mathbf{b})}\right|^{2}\right)$

Since $c_{1}(\mathbf{b})$ is the power of the estimate, $c_{1}(\mathbf{b})=\left|c_{1}(\mathbf{b})\right|$

Let us denote by * the conjugate operator, then $\left|c_{2}(\mathbf{b})\right|^{2}=c_{2}(\mathbf{b}) c_{2}{ }^{*}(\mathbf{b})$

$\frac{\delta}{\delta B}\left(\left|\frac{c_{1}(\mathbf{b})}{c_{2}(\mathbf{b})}\right|^{2}\right)=\frac{2 c_{1}(\mathbf{b}) \frac{\delta c_{1}}{\delta \mathbf{b}}(\mathbf{b})\left|c_{2}(\mathbf{b})\right|^{2}-\left|c_{1}(\mathbf{b})\right|^{2}\left[c_{2}(\mathbf{b}) \frac{\delta c_{2}{ }^{*}(\mathbf{b})}{\delta \mathbf{b}}+c_{2}{ }^{*}(\mathbf{b}) \frac{\delta c_{2}(\mathbf{b})}{\delta \mathbf{b}}\right]}{\left|c_{2}(\mathbf{b})\right|^{4}}$

$\frac{\delta}{\delta \mathbf{b}}\left(\left|\frac{c_{1}(\mathbf{b})}{c_{2}(\mathbf{b})}\right|^{2}\right)=\frac{c(\mathbf{b})}{\left|c_{2}(\mathbf{b})\right|}\left[2 \frac{\delta c_{1}(\mathbf{b})}{\delta \mathbf{b}}-\frac{c(\mathbf{b})}{\left|c_{2}(\mathbf{b})\right|}\left(c_{2}(\mathbf{b}) \frac{\delta c_{2}^{*}(\mathbf{b})}{\delta \mathbf{b}}+c_{2}^{*}(\mathbf{b}) \frac{\delta c_{2}(\mathbf{b})}{\delta \mathbf{b}}\right)\right]$

Since $c_{1}(\mathbf{b})$ and $c_{2}(\mathbf{b})$ are quadratic forms and $\mathbf{R}_{\mathbf{x}}$ as well as $\mathbf{R}_{\mathrm{x}}^{\mathbf{a}_{0}}$ are symmetric $\frac{\delta c_{1}}{\delta \mathbf{b}}(\mathbf{b})=2 \mathbf{R}_{\mathbf{x}} \mathbf{b}^{T}$ and $\frac{\delta c_{2}(\mathbf{b})}{\delta \mathbf{b}}=2 \mathbf{R}_{\mathbf{x}}^{\mathbf{a}_{0}} \mathbf{b}^{T}$

So that the gradient of the criterion is given by $\mathbf{g}(\mathbf{b})=\frac{\delta c(\mathbf{b})}{\delta \mathbf{b}}=\frac{2}{\left|c_{2}(\mathbf{b})\right|}\left[\mathbf{R}_{\mathbf{x}}-\frac{c(\mathbf{b})}{\left|c_{2}(\mathbf{b})\right|} \operatorname{real}\left\{c_{2}^{*}(\mathbf{b}) \mathbf{R}_{\mathrm{x}}^{\mathbf{a}_{0}}\right\}\right] \mathbf{b}^{T}$ 


\section{Appendix B}

\section{Hessian of the criterion}

Let us define $\mathbf{g}^{T}(\mathbf{b})=\left(\frac{\delta c(\mathbf{b})}{\delta \mathbf{b}}\right)^{T}$

The Hessian is given by $\frac{\delta \mathbf{g}^{T}(\mathrm{~b})}{\delta \mathrm{b}}$

Since $\mathbf{R}_{\mathbf{X}}$ and $\mathbf{R}_{\mathbf{X}}^{a_{0}}$ are symmetric, that is to say equal to their transposes, $\mathbf{g}^{T}(\mathbf{b})=\frac{1}{\left|c_{2}(\mathbf{b})\right|} B\left[2 \mathbf{R}_{\mathbf{x}}-\frac{c(\mathbf{b})}{\left|c_{2}(\mathbf{b})\right|}\left(c_{2}{ }^{*}(\mathbf{b}) \mathbf{R}_{\mathbf{x}}^{\mathbf{a}_{0}}+c_{2}(\mathbf{b}) \mathbf{R}_{\mathbf{\times}}^{\mathbf{a}_{0}{ }^{*}}\right)\right]$

Let us express the gradient as a sum of three function whose derivatives will be calculated separately:

$\mathbf{g}^{T}(\mathbf{b})=\mathbf{g}_{1}(\mathbf{b})+\mathbf{g}_{2}(\mathbf{b})+\mathbf{g}_{3}(\mathbf{b})$

with

$\mathbf{g}_{1}(\mathbf{b})=\frac{\mathbf{b}}{\left|c_{2}(\mathbf{b})\right|} 2 \mathbf{R}_{\mathbf{x}}$

$\mathbf{g}_{2}(\mathbf{b})=-\frac{c(\mathbf{b}) c_{2}{ }^{*}(\mathbf{b})}{\left|c_{2}(\mathbf{b})\right|^{2}} \mathbf{b} \mathbf{R}_{\times}^{\mathbf{a}_{0}}$

$\mathbf{g}_{3}(\mathbf{b})=-\frac{c(\mathbf{b}) c_{2}(\mathbf{b})}{\left|c_{2}(\mathbf{b})\right|^{2}} \mathbf{b} \mathbf{R}_{\times}^{\mathbf{a}_{0}}{ }^{*}$

Since the two matrices $\mathbf{R}_{\mathbf{x}}$ and $\mathbf{R}_{\mathbf{x}}^{\mathbf{a}_{0}}$ do not depend on $\mathbf{b}$, they can be brought into the calculation after the derivation. We finally have to calculate the derivatives of three expressions of the form $f(\mathbf{b}) \mathbf{b}$ where $f(\mathbf{b})$ equals successively to :

$$
\begin{aligned}
& f_{1}(\mathbf{b})=\frac{1}{\left|c_{2}(\mathbf{b})\right|} \\
& f_{2}(\mathbf{b})=-\frac{c(\mathbf{b}) c_{2}{ }^{*}(\mathbf{b})}{\left|c_{2}(\mathbf{b})\right|^{2}} \\
& f_{3}(\mathbf{b})=-\frac{c(\mathbf{b}) c_{2}(\mathbf{b})}{\left|c_{2}(\mathbf{b})\right|^{2}}
\end{aligned}
$$

Let us first calculate the expression of such a derivative in the more general case.

$$
\begin{aligned}
& \frac{\delta}{\delta b_{i}}(f(\mathbf{b}) \mathbf{b})=\frac{\delta}{\delta b_{i}}\left(f(\mathbf{b})\left[\begin{array}{lll}
b_{1} & \cdots & b_{P}
\end{array}\right]\right) \\
& =\frac{\delta}{\delta b_{i}}\left[\begin{array}{lll}
f(\mathbf{b}) b_{1} & \cdots & f(\mathbf{b}) b_{P}
\end{array}\right]=\left[\begin{array}{llll}
\frac{\delta}{\delta b_{i}}\left(f(\mathbf{b}) b_{1}\right) & \cdots & \frac{\delta}{\delta b_{i}}\left(f(\mathbf{b}) b_{P}\right)
\end{array}\right]
\end{aligned}
$$




$$
=\left[b_{1} \frac{\delta f(\mathbf{b})}{\delta b_{i}} \ldots\left(b_{i} \frac{\delta f(\mathbf{b})}{\delta b_{i}}+f(\mathbf{b})\right) \ldots b_{P} \frac{\delta f(\mathbf{b})}{\delta b_{i}}\right]=f(\mathbf{b}) \mathbf{i}_{i}+\left(\frac{\delta f(\mathbf{b})}{\delta b_{i}}\right) \mathbf{b}
$$

where $\mathbf{i}_{i}$ stands for the $\mathrm{i}^{\text {th }}$ row of the $P$ by $P$ identity matrix.

So that : $\frac{\delta}{\delta \mathbf{b}}(f(\mathbf{b}) \mathbf{b})=f(\mathbf{b}) \mathbf{I}+\left(\frac{\delta f(\mathbf{b})}{\delta \mathbf{b}}\right) \mathbf{b}$

Derivative of $f_{1}(B)=\frac{1}{\left|c_{2}(\mathbf{b})\right|}=\frac{1}{\sqrt{c_{2}(\mathbf{b}) C_{2}{ }^{*}(\mathbf{b})}}$

$\frac{\delta f_{1}(\mathbf{b})}{\delta \mathbf{b}}=-\frac{1}{2\left|c_{2}(\mathbf{b})\right|^{3}} \frac{\partial}{\delta \mathbf{b}}\left(c_{2}(\mathbf{b}) c_{2}^{*}(\mathbf{b})\right)$

$\frac{\delta f_{1}(\mathbf{b})}{\delta \mathbf{b}}=-\frac{1}{2\left|c_{2}(\mathbf{b})\right|^{3}}\left(c_{2}(\mathbf{b}) \frac{\delta c_{2}{ }^{*}(\mathbf{b})}{\delta \mathbf{b}}+\frac{\delta c_{2}(\mathbf{b})}{\delta \mathbf{b}} c_{2}{ }^{*}(\mathbf{b})\right)$

$\frac{\delta f_{1}(\mathbf{b})}{\delta \mathbf{b}}=-\frac{1}{2\left|c_{2}(\mathbf{b})\right|^{3}}\left(c_{2}(\mathbf{b}) \underset{\mathbf{C}}{\mathbf{g}}{ }_{2}^{*}(\mathbf{b})+\underset{\mathbf{C}}{\mathbf{g}_{2}}(\mathbf{b}) c_{2}^{*}(\mathbf{b})\right)$

where $\underset{\mathbf{C}}{\mathbf{g}}(\mathbf{b})=\frac{\delta c_{2}(\mathbf{b})}{\delta \mathbf{b}}$

Derivative of $f_{2}(\mathbf{b})=-\frac{c(\mathbf{b}) c_{2}{ }^{*}(\mathbf{b})}{\left|c_{2}(\mathbf{b})\right|^{2}}$

$\frac{\delta f_{2}(\mathbf{b})}{\delta \mathbf{b}}=-\frac{\frac{\delta\left(c(\mathbf{b}) c_{2}{ }^{*}(\mathbf{b})\right)}{\delta \mathbf{b}}\left|c_{2}(\mathbf{b})\right|^{2}-c(\mathbf{b}) c_{2}{ }^{*}(\mathbf{b}) \frac{\delta\left(\left|c_{2}(\mathbf{b})\right|^{2}\right)}{\delta \mathbf{b}}}{\left|c_{2}(\mathbf{b})\right|^{4}}$

$\frac{\delta f_{2}(\mathbf{b})}{\delta \mathbf{b}}=-\frac{1}{\left|c_{2}(\mathbf{b})\right|^{2}} \frac{\delta\left(c(\mathbf{b}) c_{2}{ }^{*}(\mathbf{b})\right)}{\delta \mathbf{b}}+\frac{c(\mathbf{b}) c_{2}{ }^{*}(\mathbf{b})\left(c_{2}(\mathbf{b}) \frac{\delta c_{2}{ }^{*}(\mathbf{b})}{\delta \mathbf{b}}+c_{2}{ }^{*}(\mathbf{b}) \frac{\delta c_{2}(\mathbf{b})}{\delta \mathbf{b}}\right)}{\mid c_{2}(\mathbf{b})^{4}}$

$\frac{\delta f_{2}(\mathbf{b})}{\delta \mathbf{b}}=-\frac{1}{\left|c_{2}(\mathbf{b})\right|^{2}}\left(\mathbf{g}(\mathbf{b}) c_{2}{ }^{*}(\mathbf{b})+c(\mathbf{b}) \mathbf{C}_{2}{ }_{2}^{*}(\mathbf{b})\right)+\frac{c(\mathbf{b}) \mathbf{g}_{2}{ }^{*}(\mathbf{b})}{\left|c_{2}{ }^{9}(\mathbf{b})\right|^{2}}+\frac{c(\mathbf{b})\left(c_{2}^{*}(\mathbf{b})\right)^{2} \mathbf{g}_{2}(\mathbf{b})}{\left|c_{2}(\mathbf{b})\right|^{4 \mathbf{C}}}$

$\frac{\delta f_{2}(\mathbf{b})}{\delta \mathbf{b}}=-\frac{\mathbf{g}(\mathbf{b}) c_{2}^{*}(\mathbf{b})}{\left|c_{2}(\mathbf{b})\right|^{2}}+\frac{c(\mathbf{b})\left(c_{2}{ }^{*}(\mathbf{b})\right)^{2} \mathbf{g}{ }_{2}(\mathbf{b})}{\left|c_{2}(\mathbf{b})\right|^{4} \mathbf{C}}$

$\frac{\delta f_{2}(\mathbf{b})}{\delta \mathbf{b}}=-\frac{\mathbf{g}(\mathbf{b})}{c_{2}(\mathbf{b})}+\frac{c(\mathbf{b}) \mathbf{g}_{2}(\mathbf{b})}{c_{2}(\mathbf{b})^{2}}$ 
Derivative of $f_{3}(\mathbf{b})=-\frac{c(\mathbf{b}) c_{2}(\mathbf{b})}{\left|c_{2}(\mathbf{b})\right|^{2}}$

We can notice that $f_{3}(\mathbf{b})=f_{2}{ }^{*}(\mathbf{b})$ and it can be shown easily that $\frac{\delta f_{3}(\mathbf{b})}{\delta \mathbf{b}}=\left(\frac{\delta f_{2}(\mathbf{b})}{\delta \mathbf{b}}\right)^{*}$

Then the Hessian can be calculated by

$\mathbf{H}(\mathbf{b})=\mathbf{H}_{1}(\mathbf{b})+\mathbf{H}_{2}(\mathbf{b})+\mathbf{H}_{3}(\mathbf{b})$

where

$\mathbf{H}_{1}(\mathbf{b})=\left[f_{1}(\mathbf{b}) \mathbf{I}+\left(\frac{\delta f_{1}(\mathbf{b})}{\delta \mathbf{b}}\right) \mathbf{b}\right] 2 \mathbf{R}_{\mathbf{x}}$

$\mathbf{H}_{2}(\mathbf{b})=\left[f_{2}(\mathbf{b}) \mathbf{I}+\left(\frac{\delta f_{2}(\mathbf{b})}{\delta \mathbf{b}}\right) \mathbf{b}\right] \mathbf{R}_{\mathbf{x}}^{\mathbf{a}_{0}}$

$\mathbf{H}_{3}(\mathbf{b})=\left[f_{3}(\mathbf{b}) \mathbf{I}+\left(\frac{\delta f_{3}(\mathbf{b})}{\delta \mathbf{b}}\right) \mathbf{b}\right] \mathbf{R}_{\mathbf{x}}^{\mathbf{a}^{*}{ }^{*}}=\mathbf{H}_{2}{ }^{*}(\mathbf{b})$

The Hessian is thus finally given by

$$
\begin{aligned}
& \mathbf{H}(\mathbf{b})=\mathbf{H}_{1}(\mathbf{b})+2 * \operatorname{real}\left\{\mathbf{H}_{2}(\mathbf{b})\right\} \\
& \begin{aligned}
\mathbf{H}(\mathbf{b})=\frac{2}{\left|c_{2}(\mathbf{b})\right|} \mathbf{R}_{\mathbf{X}} \\
+2 \operatorname{Re}\left\{-\frac{4 c_{2}^{*}(\mathbf{b})}{\left|c_{2}(\mathbf{b})\right|^{3}} \mathbf{R}_{\mathbf{X}}^{\boldsymbol{\alpha}_{0}} \mathbf{b}^{T} \mathbf{b} \mathbf{R}_{\mathbf{X}}-\frac{c(\mathbf{b}) c_{2}^{*}(\mathbf{b})}{\left|c_{2}(\mathbf{b})\right|^{2}} \mathbf{R}_{\mathbf{X}}^{\boldsymbol{\alpha}_{0}}+3\right. \\
\left.* \frac{\left(c_{2}^{*}(\mathbf{b})\right)^{2} c(\mathbf{b})}{\left|c_{2}(\mathbf{b})\right|^{4}} \mathbf{R}_{\mathbf{X}}^{\boldsymbol{\alpha}_{0}} \mathbf{b}^{\mathrm{T}} \mathbf{b} \mathbf{R}_{\mathbf{X}}^{\boldsymbol{\alpha}_{0}}+\frac{c(\mathbf{b})}{\left|c_{2}(\mathbf{b})\right|^{2}} \mathbf{R}_{\mathbf{X}}^{\boldsymbol{\alpha}_{0}{ }^{*}} \mathbf{b}^{\mathrm{T}} \mathbf{b} \mathbf{R}_{\mathbf{X}}^{\boldsymbol{\alpha}_{0}}\right\}
\end{aligned}
\end{aligned}
$$


[1] A. Ferréol, P. Chevalier, On the Behavior of Current Second and Higher Order Blind Source Separation Methods for Cyclostationary Sources, IEEE Trans. on Signal Processing 48 (6) (2000) 845-861.

[2] A. Ferréol, P. Chevalier, and L. Albera, Second-Order Blind Separation of First- and Second-Order Cyclostationary Sources - Application to AM, FSK, CPFSK, and Deterministic Sources, IEEE Trans. on Signal Processing 52 (4) (2004) 845-861. [3] P. Jallon, A. Chevreuil, Separation of instantaneous mixtures of cyclostationary sources, Signal Processing 87 (2007) 2718-2732.

[4] P. Comon, Independent component analysis, a new concept, Signal Processing 36 (3) (1994) 287-314.

[5] R. Boustany, J. Antoni, Separation of rotating machines vibration signals, twelfth International Congress on Sound and vibration, Lisbon, Portugal, 11-14 July 2005.

[6] R. Boustany, J. Antoni, A subspace method for the blind extraction of a cyclostationary source: Application to rolling element bearing diagnostics, Mechanical Systems and Signal Processing 19 (6) (2005) 1245-1259.

[7] J. Antoni, F. Guillet, M. ElBadaoui and F. Bonnardot, Blind separation of convolved cyclostationary processes, Signal Processing, 85 (2005), 51-66.

[8] S. Rhioui, N. Thirion-Moreau, E. Moreau, under-determined blind indentification of cyclostationary signals with unknown cyclic frequencies, Proc. IEEE International Conference on Acoustics, Speech and Signal Processing, Toulouse, France, 14-19 May 2006, 553-556.

[9] F. Ghaderi, B. Makkiabadi, J. McWhirter, S. Sanei, Blind source extraction of cyclostationary sources with common cyclic frequencies, Proc. IEEE International Conference on Acoustics, Speech and Signal Processing, Dallas, USA, 14-19 March 2010, 4146-4149.

[10] M. G. Jafari, S. R. Alty, J. A. Chambers, New natural gradient algorithm for cyclostationary sources, IEE proceedings. Vision, image and signal processing, 151 (1) 2004 62-68.

[11] Y. C. Liang, A. R. Leyman, B. H. Soong, Blind source separation using second order cyclic statistics, Proc. IEEE Int. Workshop on Signal Processing Advances in Wireless Communications, Paris, France, 1997, 57-60.

[12] S. Houcke, A. Chevreuil, P. Loubaton, Blind source separation of a mixture of communication sources emitting at various baud-rates, IEICE transactions on fundamentals of electronics communications and computer sciences, E86-A (3) (2003) 564-572.

[13] K. Abed-Meraim, Y. Xiang, J. H. Manton, Y. Hua, Blind source separation using second-order cyclostationary statistics, IEEE Trans. Signal Process. 49 (2) (2001) 694-701.

[14] Y. Xiang, A new algorithm for blind separation of cyclostationary source from linear mixtures, 2006 8th International Conference on Signal Processing, 16-20 November 2006, Guilin, China, 406-409. 
[15] A. Keziou, M. S . Ould Mohamed, A new criterion for second-order blind source separation, 2008 International conference on signals, circuits and systems, 79 November 2008, Hammamet, Tunisia.

[16] Y. C. Liang, A. R. Leyman, B. H. Soong, Blind source separation using second order cyclic statistics, Proc. SPAWC, Paris 1997, 57-60.

[17] R. Wang, C. Hou, D. Chen, « Blind separation of instantaneous linear mixtures of cyclostationary signals, 2010 International Conference on Image Analysis and Signal Processing, 12-14 April 2010, Xiamen, China.

[18] Y. C. Liang, A. R. Leyman, B. H. Soong, A network structure approach to blind source separation using second order cyclic statistics, IEEE international symposium on circuits and systems, 9-12 June 1997, Hong Kong.

[19] B. G. Agee, S. V. Schell, W. A. Gardner, Spectral self-coherent restoral : a new approach to blind adaptive signal extraction using antenna arrays, Proceedings of the IEEE 78 (4) (1990) 753-767.

[20] N. Bouguerriou, M. Haritopoulos, C. Capdessus, L. Allam, Novel cyclostationarity-based blind source separation algorithm using second order statistical properties : theory and application to the bearing defect diagnosis, Mechanical Systems and Signal Processing, 19 (2005), 1260-1281.

[21] R. Boustany, J. Antoni, A subspace method for the blind extraction of a cyclostationary source : application to rolling element bearing diagnostics, Mechanical Systems and Signal Processing, 19 (2005), 1245-1259.

[22] F. Ghaderi, H. R. Mohseni, J. G. McWhirter, S. Sanei, Blind source extraction of periodic signals, Proc. IEEE International Conference on Acoustics, Speech and Signal Processing, 19-24 April 2009, Taipei, Taiwan, 377-380.

[23] Y. Xiang, A new algorithm for blind separation of cyclostationary sources from linear mixtures, 8th International Conference on Signal Processing, 16-20 November 2006, Guilin, China.

[24] C. Capdessus, A. K. Nandi, N. Bouguerriou, A New Source Extraction Algorithm for Cyclostationary Sources, 7th International Conference on Independent Component Analysis and Signal Separation, London, UK, 9 - 12 September 2007, 145-151.

[25] C. Capdessus, A. K. Nandi, N. Bouguerriou, Source extraction algorithm based on cyclic properties, Independent Component Analysis Research Network International Workshop, Liverpool, UK, 25-26 September 2008, 36-39.

[26] M. Haritopoulos, C. Capdessus, A. K. Nandi, Foetal PQRST Extraction from ECG Recordings Using Cyclostationarity-Based Source Separation Method, 32nd Annual International Conference of the IEEE Engineering in Medicine and Biology Society, August 31 - September 4, 2010, Buenos Aires, Argentina.

[27] M. Haritopoulos, C. Capdessus and A. K. Nandi, Extraction of Foetal Contribution to ECG Recordings Using Cyclostationarity-Based Source Separation Method, Ninth International Conference on Latent Variable Analysis and Signal Separation, September 27-30, Saint-Malo, France.

[28] A. Antoniou, W. S. Lu, Practical Optimization, Algorithms and Engineering Applications, Springer, 2007. 